\title{
Angular Dependence of Spin Transfer Switching in Spin Valve Nanopillar Based Heusler Alloy
}

\author{
Pirat Khunkitti, ${ }^{1}$ Anan Kruesubthaworn, ${ }^{1}$ Arkom Kaewrawang, ${ }^{1}$ Tim Mewes, \\ Claudia K. A. Mewes, ${ }^{2}$ and Apirat Siritaratiwat ${ }^{1}$ \\ ${ }^{1}$ KKU-Seagate Cooperation Research Laboratory, Department of Electrical Engineering, Faculty of Engineering, \\ Khon Kaen University, Khon Kaen 40002, Thailand \\ ${ }^{2}$ Department of Physics and Astronomy, MINT Center, University of Alabama, Tuscaloosa, AL 35487, USA
}

Correspondence should be addressed to Apirat Siritaratiwat; apirat@kku.ac.th

Received 1 December 2015; Revised 14 April 2016; Accepted 3 May 2016

Academic Editor: Santiago Garcia-Granda

Copyright (C) 2016 Pirat Khunkitti et al. This is an open access article distributed under the Creative Commons Attribution License, which permits unrestricted use, distribution, and reproduction in any medium, provided the original work is properly cited.

\begin{abstract}
The spin transfer induced magnetization switching in current perpendicular-to-the-plane spin valve nanopillar based $\mathrm{Co}_{2} \mathrm{FeAl}_{0.5} \mathrm{Si}_{0.5}$ Heusler alloy with varying the initial angles of the magnetization of sensing layer, $\theta_{0}$, was investigated via macrospin simulations. The effects of an in-plane magnetic field, $H_{i}$, on the switching behavior were also evaluated. The magnetization switching was excited by spin polarized switching current, $I_{s}$. The time varying magnetization was computed by the LandauLifshitz-Gilbert-Slonczewski equation, while the spin transfer induced noise was examined by using the power spectral density analysis. It was found that $\theta_{0}$ should be narrowly initialized since this configuration produces the small noise during the switching. Also, the negative $I_{s}$ produced more uniform switching than the positive $I_{s}$ due to existence of ferromagnetic exchange coupling. When $H_{i}$ was presented, the noise generated at low frequencies could be suppressed, and then the switching behavior became more uniform. In addition, the results indicated that the noise configuration could be explained by the physical dynamic of magnetization behavior. Hence, the spin transfer induced noise needs to be minimized in order to improve the performance of spin transfer torque random access memory for high density recording.
\end{abstract}

\section{Introduction}

In 1996, Slonczewski discovered that a spin polarized current could transfer the angular momentum to the magnetization of ferromagnet by spin transfer torque phenomenon, which has become the major mechanism of spin transfer torque random access memory (STT-RAM) [1, 2]. Also, the STT-RAM has been extensively proposed to be the memory for high density recording technologies because of its outstanding performances including high operating speed, high reliability, long endurance, nonvolatility, and low writing energy [3-6]. Then, an improvement of the STT-RAM performance by reducing the switching current has been of wide interest in order to minimize the energy consumption and increase the operating speed $[5,7-9]$. In particular, the important factors which directly impact the switching current and the switching behavior were the initial angle between the magnetization of free and pinned layers, $\theta_{0}$, and the external magnetic field [10-12].

Nowadays, the ferromagnetic Heusler alloy materials are of wide interest for the spintronic applications due to their various advantages such as high spin polarization, high MR ratio, high Curie temperature, and high magnetic moment [13-16]. Particularly, the spin valve using $\mathrm{Co}_{2} \mathrm{FeAl}_{0.5} \mathrm{Si}_{0.5}$ (CFAS) Heusler alloy as the ferromagnetic electrode presented a high MR ratio of $6.9 \%$ with Ag spacer [17]. Several researches have focused on an enhancement of MR ratio of CFAS Heusler alloy; however, the spin transfer switching in the spin valve nanopillars based on this alloy has been rarely presented [18].

Hence, the aim of this paper is to investigate the spin transfer induced magnetization switching in the spin valve nanopillar based on CFAS Heusler alloy with varying initial angle, $\theta_{0}$, including the effects of the external 


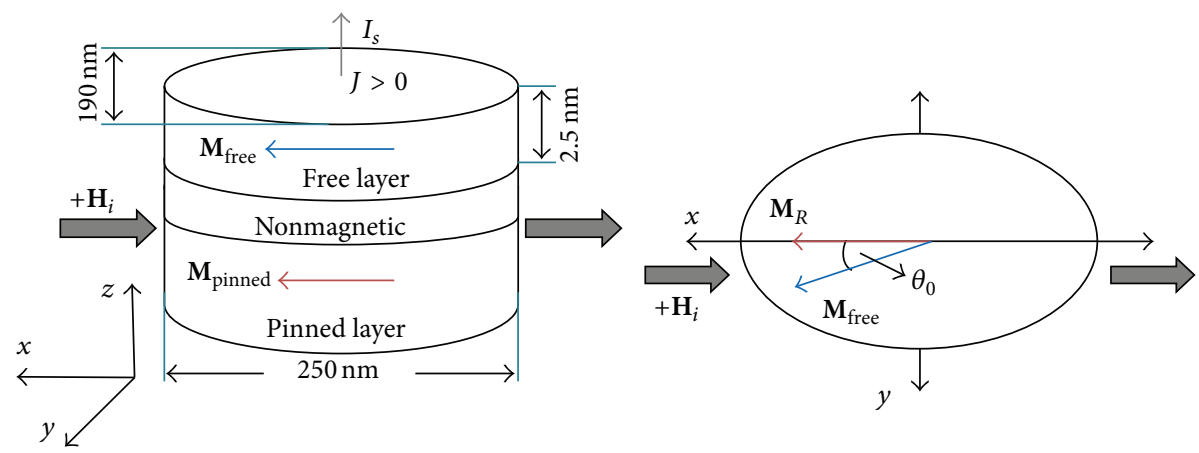

FIGURE 1: Geometric definition of spin valve nanopillar based on CFAS Heusler alloy.

in-plane magnetic field, $H_{i}$. The macrospin model was performed in the simulations based on Landau-Lifshitz-GilbertSlonczewski equation by using MATLAB code $\mathrm{M}^{3}$ program [19].

\section{Simulation Model}

The simulation model of spin valve nanopillar is an elliptical geometry with a cross section area of $250 \times 190 \mathrm{~nm}^{2}$, as shown in Figure 1. It consists of the pinned and free layers separated by a nonmagnetic layer which are CFAS $(20 \mathrm{~nm}) / \mathrm{Ag}$ $(4 \mathrm{~nm}) /$ CFAS $(2.5 \mathrm{~nm})$ [18]. The magnetization of free and pinned layers is initially aligned along $x$-axis which is its easy axis. Also, the magnetization dynamic under spin polarized current is generally computed by Landau-Lifshitz-Gilbert equation including a spin transfer torque term, written as follows [20]:

$$
\begin{aligned}
\frac{\mathrm{d} \mathbf{M}}{\mathrm{d} t}= & -\gamma \mathbf{M} \times \mathbf{H}_{\mathrm{eff}}-\alpha \frac{\gamma}{M_{s}} \mathbf{M} \times\left(\mathbf{M} \times \mathbf{H}_{\mathrm{eff}}\right)-a_{j} \frac{\gamma}{M_{s}} \mathbf{M} \\
& \times\left(\mathbf{M} \times \mathbf{m}_{p}\right) .
\end{aligned}
$$

Equation (1) consists of precession term, damping term, and spin transfer torque term, respectively. $\mathbf{M}$ and $\mathbf{m}_{p}$ are the magnetization of the free layer and the direction of electron polarization, respectively. $\gamma, \alpha$, and $M_{s}$ are gyromagnetic ratio, Gilbert damping constant, and saturation magnetization, respectively. $\mathbf{H}_{\mathrm{eff}}$ is the effective magnetic field including the anisotropy field, exchange field, demagnetization field, and external field. The spin torque factor, $a_{j}$, is defined as follows:

$$
a_{j}=\frac{\hbar}{2 e} \frac{g(\theta)}{M_{s} \delta} J
$$

where $g(\theta)$ is the scalar function written as $g(\theta)=$ $\left[-4+(1+p)^{3}(3+\cos \theta) / 4 p^{3 / 2}\right]^{-1}, \theta$ is the angle between the magnetization of free layer and pinned layers, $p$ is the spin polarization, $\hbar=h / 2 \pi$, where $h$ is Planck's constant, $J$ is the spin current density, $e$ is the absolute value of electron charge, and $\delta$ is the thickness of the free layer.

The CFAS Heusler alloy has the following magnetic parameters [18]: $M_{s}$ of $9.0 \times 10^{5} \mathrm{~A} / \mathrm{m}, \alpha$ of $0.01, p$ of
0.76, exchange stiffness constant, $A$, of $2.0 \times 10^{-11} \mathrm{~J} / \mathrm{m}$, and magnetocrystalline anisotropy constant, $K_{1}$, of $-1.0 \times$ $10^{4} \mathrm{~J} / \mathrm{m}^{3}$. The system was relaxed before the magnetization switching was excited by a constant switching current, $I_{s}$, of two times of the critical current. This was because this magnitude produced a minimum switching energy during the switching process [21]. The positive $I_{s}$ is generally defined as the electron flowing from the free layer to the pinned layer which causes the magnetization switching from parallel (P) to antiparallel (AP) state. On the other hand, negative $I_{s}$ is defined as the electron flowing from the pinned layer to free layer, resulting in the magnetization reversal from AP to $\mathrm{P}$ state. In this investigation, the magnetization of free layer was initialized along $+x$ and $-x$ directions for cases using the positive and negative $I_{s}$, respectively.

In this study, the noise power spectral density (PSD) generated during the switching was investigated with $\theta_{0}$ of $0^{\circ}$ to $30^{\circ}$. Furthermore, in order to evaluate the effects of $H_{i}$ on the noise PSD, the constant $H_{i}$ of $3,980 \mathrm{~A} / \mathrm{m}$ (50 Oe) was applied along $-x$ and $+x$ directions for the cases using the positive and the negative $I_{s}$, respectively.

First of all, the magnetization switching time, $\tau_{\text {sw }}$, of spin transfer switching with varying $\theta_{0}$ was evaluated. $\tau_{\mathrm{sw}}$ was defined as the time period of magnetization oscillation starting from the initial state until the magnetization crosses $y$-axis which is perpendicular to the initial easy axis. Then, the noise PSD generated from the spin transfer switching was further considered by using the PSD analysis.

The local PSD of the magnetization dynamic was firstly computed by the discrete Fourier transform of the local time varying magnetization, $\mathbf{M}_{x, y, z}\left(\mathbf{r}_{i}, t_{k}\right)$, for each point, $\mathbf{r}_{i}$, and each time, $t_{k}$, as written in the following equation:

$$
S_{x, y, z}\left(\mathbf{r}_{i}, f\right)=\left|\sum_{i} \mathbf{M}_{x, y, z}\left(\mathbf{r}_{i}, t_{k}\right) e^{j 2 \pi f t_{k}}\right|^{2} .
$$

Accordingly, the overall PSD was calculated by summation of local PSD of the time varying magnetization, given as the following equation [22]:

$$
\bar{S}_{x, y, z}(f)=\sum_{i} S_{x, y, z}\left(\mathbf{r}_{i}, f\right) .
$$




\section{Results and Discussions}

Figure 2 illustrates $\tau_{\mathrm{sw}}$ with varying $\theta_{0}$, resulting from the positive and negative $I_{s}$, including the effects of $H_{i}$. Figures 2(a)-2(f) show the examples of the magnetization switching behavior projected in $x, y$, and $z$ directions for each particular point. From the simulation results in Figure 2, it is indicated that $\tau_{\mathrm{sw}}$ is reduced either by increasing $\theta_{0}$ or applying $H_{i}$. The dependence of $\tau_{\text {sw }}$ on $\theta_{0}$ can be clearly seen from a comparison between Figures 2(a) and 2(b) and between Figures 2(e) and 2(f). The dependence of $\tau_{\text {sw }}$ on $H_{i}$ is shown by a comparison between Figures 2(a), 2(c), and 2(e) and between Figures 2(b), 2(d), and 2(f). Also, negative $I_{s}$ causes shorter $\tau_{\text {sw }}$ than positive $I_{s}$, as can be seen from a comparison between Figures 2(c) and 2(d) and between Figures 2(a) and 2(b). These results could be explained by using (1); that is, an increase of $\theta_{0}$ yields an enhancement of $g(\theta)$ which results in a large $a_{j}$. Then, large $a_{j}$ further causes a massive spin transfer torque term which leads to a reduction of $\tau_{\text {sw }}$ [20]. Likewise, applying $H_{i}$ decreases the magnitude of $\mathbf{H}_{\text {eff }}$ which also reduces the damping term. Then, a small damping term brings about short $\tau_{\mathrm{sw}}$ because the magnetization could be easily switched into the reversal. Additionally, negative $I_{s}$ results in shorter $\tau_{\mathrm{sw}}$ than positive $I_{s}$ because of an asymmetry of the critical current for switching, $I_{c}$, which is generally proportional to $\tau_{\mathrm{sw}}$ [21]. It is indicated that $I_{c}{ }^{\mathrm{AP} \rightarrow \mathrm{P}}$ is always lower than $I_{c} \mathrm{P} \rightarrow \mathrm{AP}$ due to an asymmetry of $g(\theta)$, as described by Mangin et al. [23].

For the investigation of the noise PSD generated during the switching, the time varying magnetization in $y$ direction is focused. This is because the overall PSD is mainly generated from the magnetization oscillation in $y$ direction which also produces the resonance frequency of the magnetization switching [12].

Figure 3 illustrates the noise PSD generated from positive $I_{s}$ (Figure 3(a)) and negative $I_{s}$ (Figure 3(b)) with varying $\theta_{0}$. From Figure 3(a), it is shown that the PSD has a minimum value at $\theta_{0}=1^{\circ}$ which is indicated as the smallest noise generated in the switching process. After that, the PSD tends to increase by an increment of $\theta_{0}$ from $1^{\circ}$ to $20^{\circ}$ and it decreases afterwards when $\theta_{0}$ is increased up to $30^{\circ}$. The changes of PSD configuration could be described by the physical behavior of time varying magnetization. The amplitude of PSD is generally proportional to the magnitude and time periods of the magnetization oscillation, whereas the frequency of PSD depends on the magnetization oscillating frequency. The examples of magnetization switching behavior are displayed in Figures 2(a)-2(f). By a consideration of time varying magnetization, it is found that the PSD generated at low frequency components (less than $4 \mathrm{GHz}$ ) mainly result from the magnetization oscillation during the switching period. In this work, the switching period is defined as the period when the magnetization is tiled more than $30^{\circ}$ out of an easy axis approximately. In contrast, the PSD generated at high frequency components (higher than $4 \mathrm{GHz}$ ) are generated from the magnetization oscillation at the time period before and after switching period. During the switching period, the magnetization becomes the most unstable because it is highly aligned out of its easy axis which is quite unstable configuration. Then, the noise could be extremely generated during the switching period. The results also show that the PSD at low frequency components are higher than high frequency components, which implies that the noise generated during the switching period plays a significant role in the overall noise PSD. Thus, the changes of the PSD configurations at low and high frequency components are proportional to the variations of magnetization oscillation during the switching period and before and after switching period, respectively. On the other hand, Figure 3(b) shows that the magnitude of the PSD generated by negative $I_{s}$ is insignificantly changed with varying $\theta_{0}$. This is because negative $I_{s}$ causes slight differences of the magnetization oscillation when $\theta_{0}$ is varied, which brings about the little changes of $\tau_{\mathrm{sw}}$, as can be seen from Figure 2(d) compared to Figure 2(c). Also, negative $I_{s}$ results in two obvious peaks of PSD located at low and high frequency components. The low frequency peak is located at about $3 \mathrm{GHz}$, whereas the high frequency peak is located at about $4.7 \mathrm{GHz}$. In this case, it is found that the PSD peak at low frequency is induced by the magnetization oscillation during the switching period, whereas the high frequency peak is excited by magnetization oscillation at preswitching and postswitching period. In addition, a comparison between Figures 3(a) and 3(b) indicates that negative $I_{s}$ produces the lower and more uniform PSD than positive $I_{s}$. This is because negative $I_{s}$ causes the AP to $\mathrm{P}$ switching which is more uniform than the P to AP switching induced by positive $I_{s}$, which could be described by the behavior of an exchange coupling interaction. The exchange coupling is an interaction that tries to maintain the stable state between two nearby spins, which can imply the stability of the magnetization orientation between two adjacent layers. Also, this interaction directly affects the exchange energy of ferromagnetic system under the magnetic field as given in the following equation $[23,24]$ :

$$
E_{\text {exchange }}=A_{\mathrm{ex}} \int(\nabla \mathrm{m})^{2} d v
$$

where $m=M / M_{s}$ is the normalized magnetization vector, $v$ is the volume of material, and $A_{\mathrm{ex}}=\left(n J_{\mathrm{ex}} S^{2} / a\right)$ is the exchange stiffness constant, where $n$ is the number of atoms per unit cell, $S$ is the spin, $a$ is the lattice parameter, and $J_{\mathrm{ex}}$ is the exchange integral which indicates the types of coupling by its sign. The ferromagnetic coupling occurs with the positive sign of $J_{\text {ex }}$, whereas the antiferromagnetic coupling occurs when the sign of $J_{\mathrm{ex}}$ is negative. The exchange energy represents the existent energy of the magnetic domain wall configuration [24]. This energy indicates the stability of the magnetization alignment between two adjacent layers stabilized by an exchange force. The maximum stability of two nearby magnetizations occurs with the minimum exchange energy. For the ferromagnetic coupling, the maximum stability occurs when the magnetizations of two adjacent layers are parallel. On the other hand, the maximum stability of the antiferromagnetic coupling occurs when two neighbor magnetizations are antiparallel. In this model, a positive sign of $J_{\mathrm{ex}}$ indicates the ferromagnetic coupling which becomes stable when magnetization of free layer and magnetization of pinned layer are parallel. Then, the AP to P switching caused 


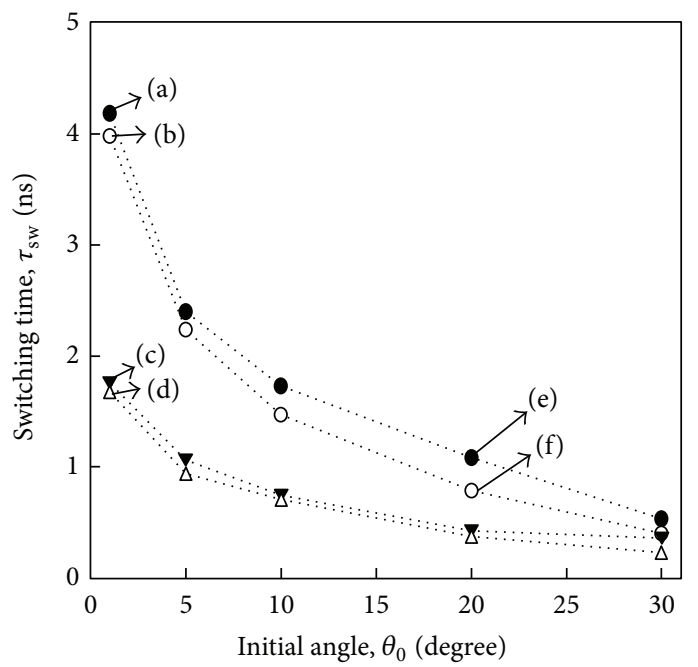

-. Positive $I_{s}$

$\nabla$. Negative $I_{s}$

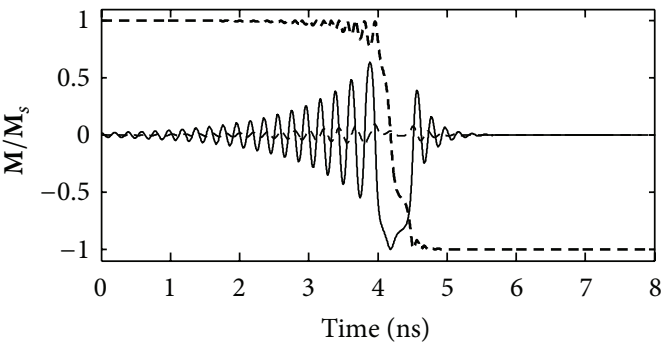

(a)

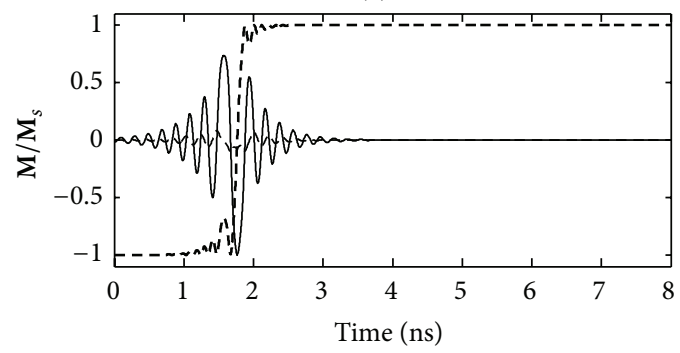

(c)

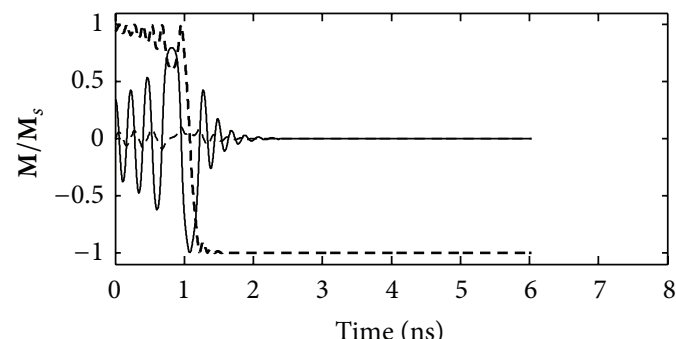

-. $\mathbf{M}_{x}$

$-\mathbf{M}_{y}$

-.- $\mathbf{M}_{z}$
O. Positive $I_{s}$ with $H_{i}$

$\triangle$. Negative $I_{s}$ with $H_{i}$

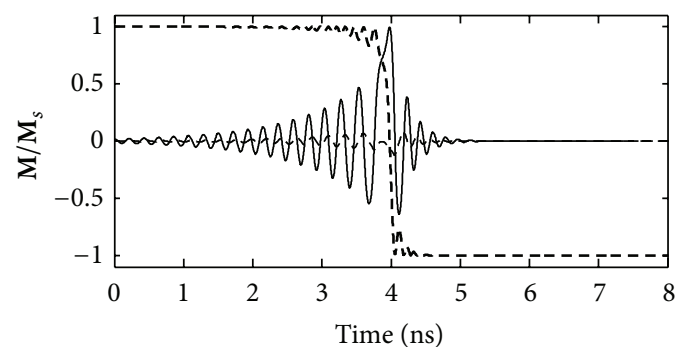

(b)

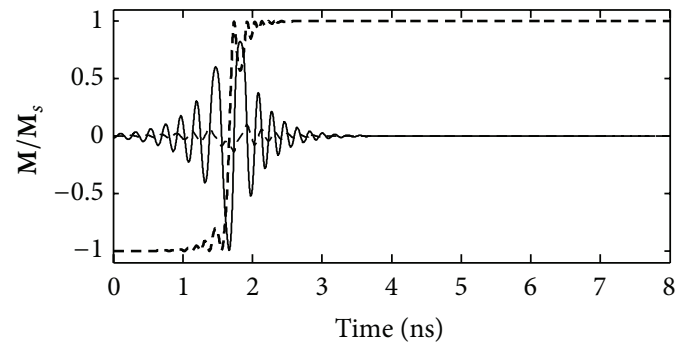

(d)

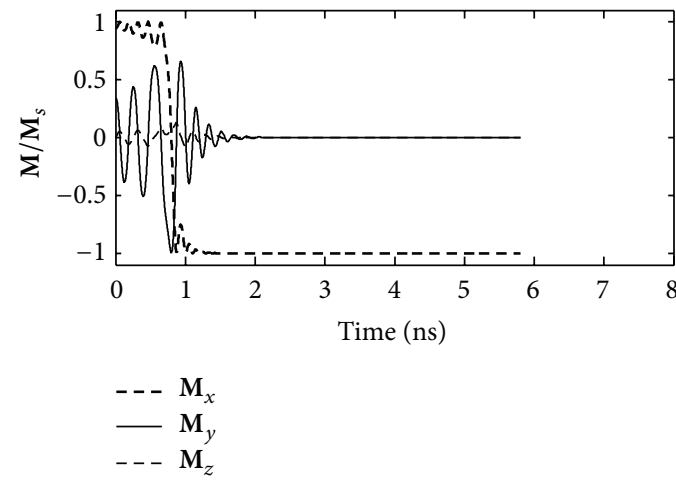

(f)

(e)

FIGURE 2: Magnetization switching time, $\tau_{\mathrm{sw}}$, with varying $\theta_{0}$, resulting from the positive and negative $I_{s}$, including the effects of $H_{i}$. (a) and (b): the magnetization switching behavior resulting from positive $I_{s}$ at $\theta_{0}=1^{\circ}$ without and with an effect of $H_{i}$, respectively. (c) and (d): the magnetization switching behavior resulting from negative $I_{s}$ at $\theta_{0}=1^{\circ}$ without and with an effect of $H_{i}$, respectively. (e) and (f): the magnetization switching behavior caused by positive $I_{s}$ at $\theta_{0}=20^{\circ}$ without and with an effect of $H_{i}$, respectively. 


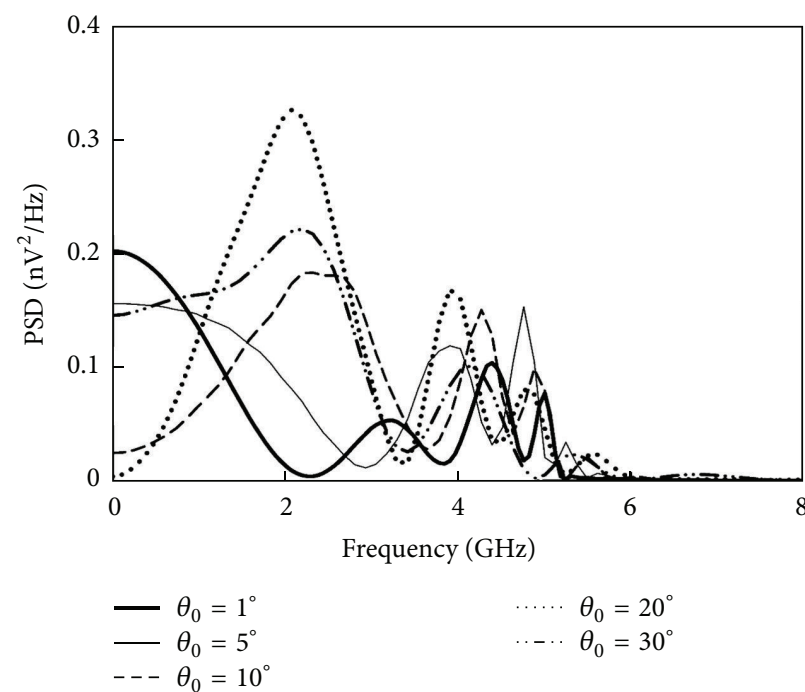

(a)

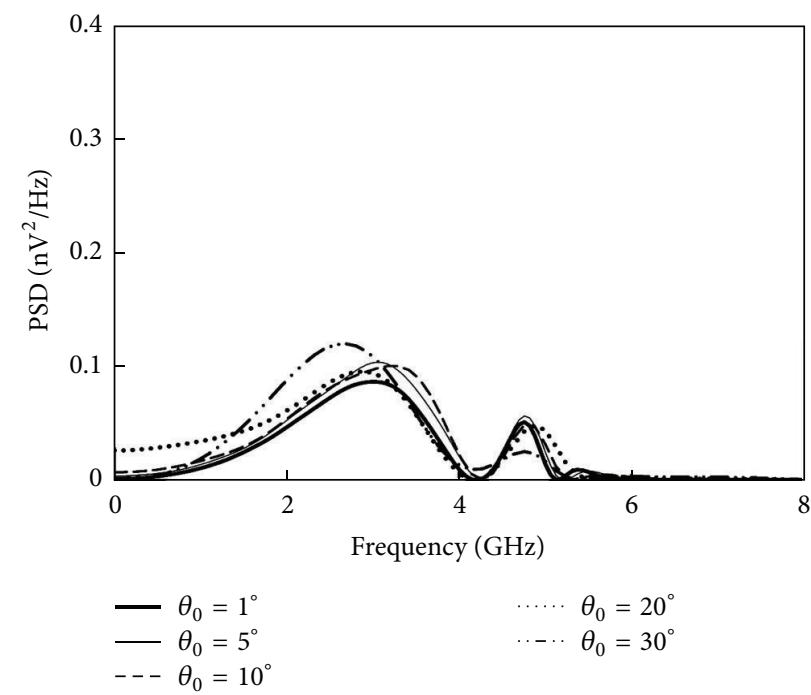

(b)

FIGURE 3: Noise PSD of spin transfer switching for different $\theta_{0}$, generated from (a) positive $I_{s}$ and (b) negative $I_{s}$.

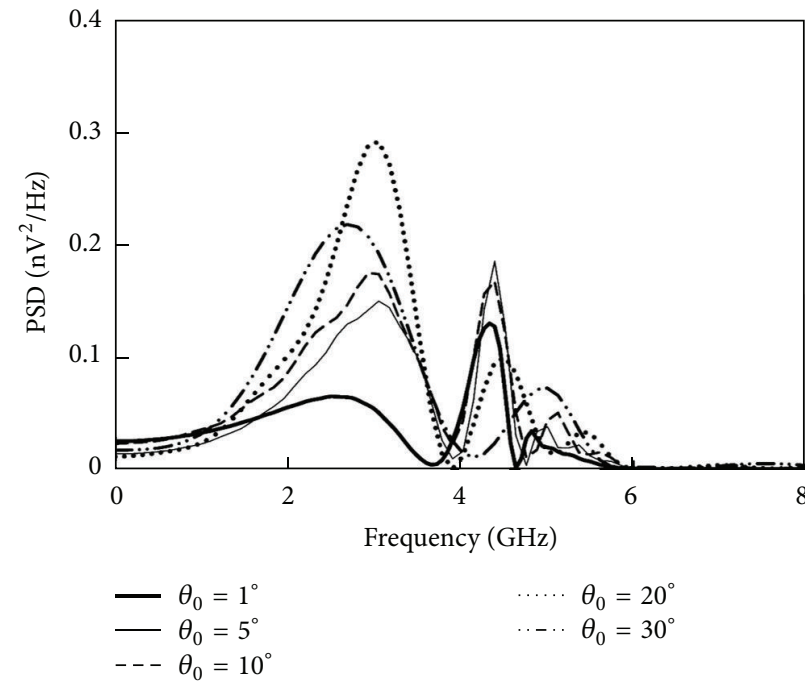

(a)

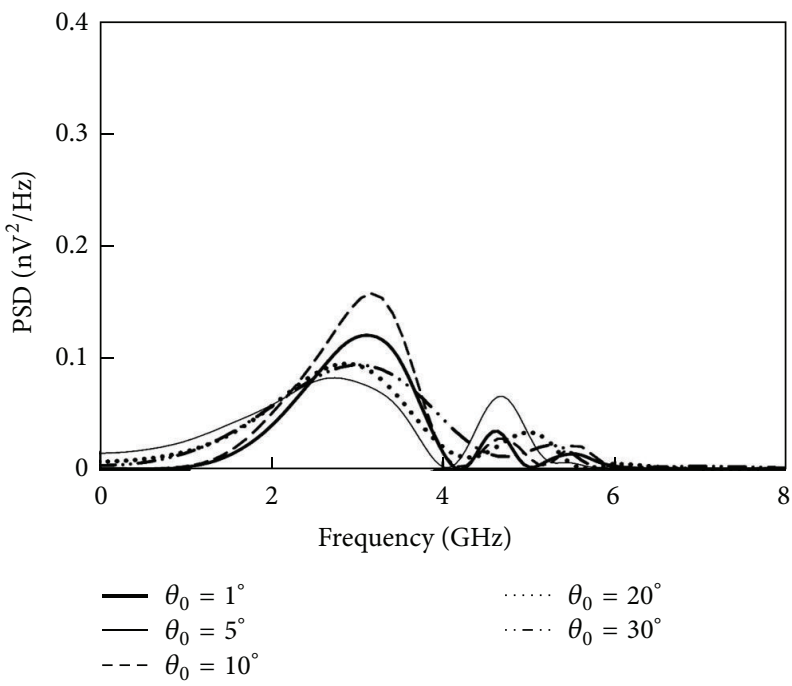

(b)

FIGURE 4: Noise PSD of spin transfer switching for different $\theta_{0}$ with applying $H_{i}$, generated from (a) positive $I_{s}$ and (b) negative $I_{s}$.

by negative $I_{s}$ could be reached with lower noise and more uniform response than the P to AP switching induced by positive $I_{s}$.

Furthermore, Figures 4(a) and 4(b) show the PSD of the magnetization switching including an effect of $H_{i}$ with using positive and negative $I_{s}$, respectively. It is found that the PSD produced by positive $I_{s}$ is divided into low and high frequency components, as displayed in Figure 4(a). For the low frequency component, the PSD starts to increase from a minimum value at $\theta_{0}=1^{\circ}$. Then, the PSD is continually enhanced when $\theta_{0}$ is raised up to $20^{\circ}$ and it is decreased afterwards with increasing $\theta_{0}$ to $30^{\circ}$, which is similar to the changes of PSD for a case without $H_{i}$. Nevertheless, the high frequency component is enlarged only when $\theta_{0}$ increases from $1^{\circ}$ to $5^{\circ}$; after that it is reduced with increasing $\theta_{0}$.
Also, the peak at low frequency is higher than the peak at high frequency, similar to that found in a case without $H_{i}$. Similar to the PSD for a case without applying $H_{i}$, a comparison between Figures 4(a) and 4(b) indicates that the PSD can be reduced by using negative $I_{s}$. In particular, it is discovered that the noise PSD at frequencies lower than $1 \mathrm{GHz}$ is suppressed by applying $H_{i}$, which can be clearly seen from Figure 4(a) compared to Figure 3(a). This is because $H_{i}$ forces the magnetization into the reversal which can reduce the switching time and also the switching period, as illustrated in Figures 2(b), 2(d), and 2(f) compared to Figures 2(a), 2(c), and 2(e). As explained in Figure 3, the noise at frequency range between 0 and $4 \mathrm{GHz}$ is mainly induced during the switching period, and then applying $H_{i}$ can suppress the noise generated at frequencies less than 
$1 \mathrm{GHz}$ because some periods of the switching period which produce the noise at this frequency range are reduced. Thus, the magnetization reversal becomes more uniform with two separated peaks when $H_{i}$ is presented, which benefits in terms of design because the frequency response of the system is less complicated. From the simulation results, $\theta_{0}$ can be set by applying the external magnetic field to the magnetization of free layer in the particular direction. As a result, the magnetization of free layer can be initially aligned along the given direction. Hence, the results suggested that the spin transfer switching should be initialized with $\theta_{0}=1^{\circ}$ including the effect of $H_{i}$ in order to minimize the instabilities generated during the switching.

\section{Conclusions}

The spin transfer induced magnetization switching in spin valve nanopillar with varying $\theta_{0}$, including the effects of $H_{i}$, was investigated. It was found that the magnetization of free and pinned layers initialized with a small $\theta_{0}$ results in a small PSD generated during switching. Also, the negative $I_{s}$ creates a more uniform reversal than the positive $I_{s}$. In addition, the dependence of the PSD on the physical dynamics of magnetization switching was studied in detail. It was indicated that the magnetization oscillation during the switching period plays the most significant role to the overall PSD. Furthermore, the results confirmed that including an external field, $H_{i}$, can significantly reduce the switching times and the PSD, while at the same time it tends to lead to a more uniform response. As a result of our calculations, the frequency response of the system can be parametrized which can be beneficial to design the spin valves in terms of avoiding the interference from the spin transfer induced reversals.

\section{Competing Interests}

The authors declare that they have no competing interests.

\section{Acknowledgments}

This work was financially supported by the Thailand Research Fund through the Royal Golden Jubilee Ph.D. Program (Grant no. PHD/0103/2554) to Mr. Pirat Khunkitti and Professor Dr. Apirat Siritaratiwat.

\section{References}

[1] J. C. Slonczewski, "Current-driven excitation of magnetic multilayers," Journal of Magnetism and Magnetic Materials, vol. 159, no. 1-2, pp. L1-L7, 1996.

[2] L. Berger, "Emission of spin waves by a magnetic multilayer traversed by a current," Physical Review B-Condensed Matter and Materials Physics, vol. 54, no. 13, pp. 9353-9358, 1996.

[3] T. Kawahara, K. Ito, R. Takemura, and H. Ohno, "Spin-transfer torque RAM technology: review and prospect," Microelectronics Reliability, vol. 52, no. 4, pp. 613-627, 2012.

[4] Z. Jian-Gang, "Magnetoresistive random access memory: the path to competitiveness and scalability," Proceedings of the IEEE, vol. 96, no. 11, pp. 1786-1798, 2008.
[5] H. Zhao, A. Lyle, Y. Zhang et al., "Low writing energy and sub nanosecond spin torque transfer switching of in-plane magnetic tunnel junction for spin torque transfer random access memory," Journal of Applied Physics, vol. 109, no. 7, Article ID 07C720, 2011.

[6] C. W. Smullen, V. Mohan, A. Nigam, S. Gurumurthi, and M. R. Stan, "Relaxing non-volatility for fast and energy-efficient STTRAM caches," in Proceedings of the 17th International Symposium on High-Performance Computer Architecture (HPCA '11), pp. 50-61, San Antonio, Tex, USA, February 2011.

[7] R. Sbiaa, S. Y. H. Lua, R. Law, H. Meng, R. Lye, and H. K. Tan, "Reduction of switching current by spin transfer torque effect in perpendicular anisotropy magnetoresistive devices," Journal of Applied Physics, vol. 109, no. 7, Article ID 07C707, 2011.

[8] P. Baláž, M. Gmitra, and J. Barnaś, "Current-pulse-induced magnetic switching in standard and nonstandard spin-valves: theory and numerical analysis," Physical Review B-Condensed Matter and Materials Physics, vol. 79, no. 14, Article ID 144301, 2009.

[9] P. K. Amiri, Z. M. Zeng, P. Upadhyaya et al., "Low write-energy magnetic tunnel junctions for high-speed spin-transfer-torque MRAM," IEEE Electron Device Letters, vol. 32, no. 1, pp. 57-59, 2011.

[10] N. Smith, J. A. Katine, J. R. Childress, and M. J. Carey, "Angular dependence of spin torque critical currents for CPP-GMR read heads," IEEE Transactions on Magnetics, vol. 41, no. 10, pp. 29352940, 2005.

[11] J. Barnaś, A. Fert, M. Gmitra, I. Weymann, and V. K. Dugaev, "From giant magnetoresistance to current-induced switching by spin transfer," Physical Review B, vol. 72, no. 2, Article ID 024426, 2005.

[12] C.-M. Lee, J.-S. Yang, and T.-H. Wu, "Micromagnetic simulation for spin-transfer switching with a tilted spin polarizer," IEEE Transactions on Magnetics, vol. 47, no. 3, pp. 649-652, 2011.

[13] R. Shan, H. Sukegawa, W. H. Wang et al., "Demonstration of half-metallicity in fermi-level-tuned heusler alloy $\mathrm{Co}_{2} \mathrm{FeAl}_{0.5} \mathrm{Si}_{0.5}$ at room temperature," Physical Review Letters, vol. 102, no. 24, Article ID 246601, 2009.

[14] T. M. Nakatani, N. Hase, H. S. Goripati, Y. K. Takahashi, T. Furubayashi, and K. Hono, "Co-based Heusler alloys for CPPGMR spin-valves with large magnetoresistive outputs," IEEE Transactions on Magnetics, vol. 48, no. 5, pp. 1751-1757, 2012.

[15] M. Shaughnessy, R. Snow, L. Damewood, and C. Y. Fong, "Memory and spin injection devices involving half metals," Journal of Nanomaterials, vol. 2011, Article ID 140805, 6 pages, 2011.

[16] T. M. Nakatani, T. Furubayashi, and K. Hono, "Interfacial resistance and spin-dependent scattering in the currentperpendicular-to-plane giant magnetoresistance using $\mathrm{Co}_{2} \mathrm{Fe}\left(\mathrm{Al}_{0.5} \mathrm{Si}_{0.5}\right)$ Heusler alloy and Ag," Journal of Applied Physics, vol. 109, no. 7, Article ID 07B724, 2011.

[17] T. Furubayashi, K. Kodama, H. Sukegawa, Y. K. Takahashi, K. Inomata, and K. Hono, "Current-perpendicular-to-plane giant magnetoresistance in spin-valve structures using epitaxial $\mathrm{Co}_{2} \mathrm{FeAl}_{0.5} \mathrm{Si}_{0.5} / \mathrm{Ag} / \mathrm{Co}_{2} \mathrm{FeAl}_{0.5} \mathrm{Si}_{0.5}$ trilayers," Applied Physics Letters, vol. 93, no. 12, Article ID 122507, 2008.

[18] H. B. Huang, X. Q. Ma, Z. H. Liu et al., "Micromagnetic simulation of spin-transfer switching in a full-Heusler $\mathrm{Co}_{2} \mathrm{FeAl}_{0.5} \mathrm{Si}_{0.5}$ alloy spin-valve nanopillar," Journal of Applied Physics, vol. 110, no. 3, Article ID 033913, 2011. 
[19] C. K. A. Mewes and T. Mewes, "Matlab based micromagnetics code M3," http://www.bama.ua.edu/ tmewes/Mcube/Mcube .shtml.

[20] D. C. Ralph and M. D. Stiles, "Spin transfer torques," Journal of Magnetism and Magnetic Materials, vol. 320, no. 7, pp.1190-1216, 2008.

[21] C. Surawanitkun, A. Kaewrawang, A. Siritaratiwat et al., "Modeling of switching energy of magnetic tunnel junction devices with tilted magnetization," Journal of Magnetism and Magnetic Materials, vol. 381, pp. 220-225, 2015.

[22] R. D. McMichael and M. D. Stiles, "Magnetic normal modes of nanoelements," Journal of Applied Physics, vol. 97, no. 10, Article ID 10J901, 2005.

[23] S. Mangin, D. Ravelosona, J. A. Katine, M. J. Carey, B. D. Terris, and E. E. Fullerton, "Current-induced magnetization reversal in nanopillars with perpendicular anisotropy," Nature Materials, vol. 5, no. 3, pp. 210-215, 2006.

[24] R. Hu, A.-K. Soh, G.-P. Zheng, and Y. Ni, "Micromagnetic modeling studies on the effects of stress on magnetization reversal and dynamic hysteresis," Journal of Magnetism and Magnetic Materials, vol. 301, no. 2, pp. 458-468, 2006. 

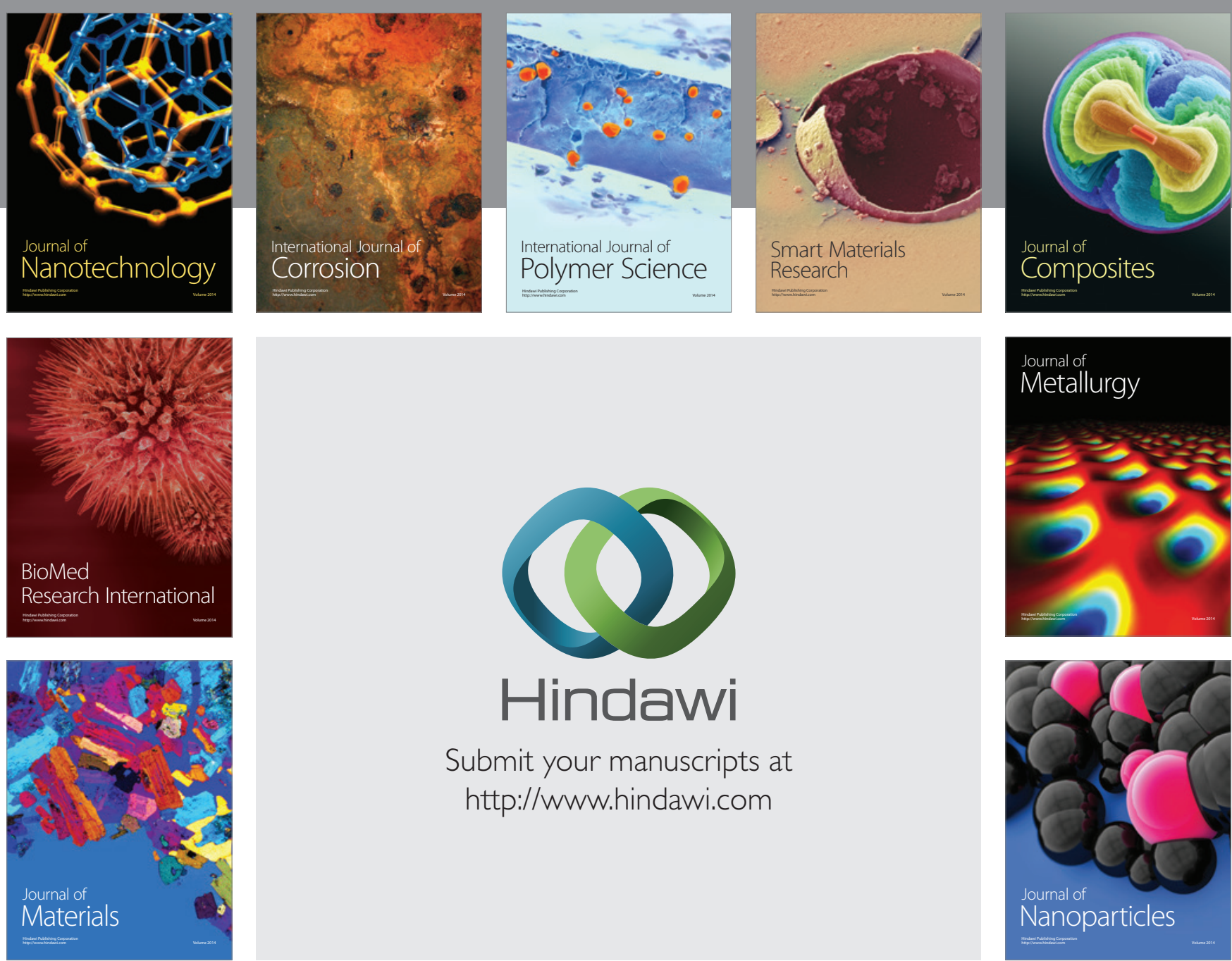

\section{Hindawi}

Submit your manuscripts at

http://www.hindawi.com

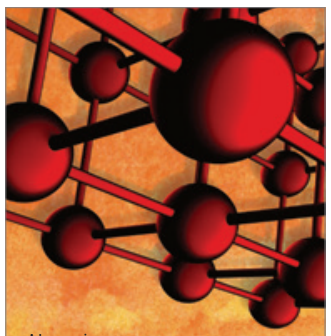

Materials Science and Engineering
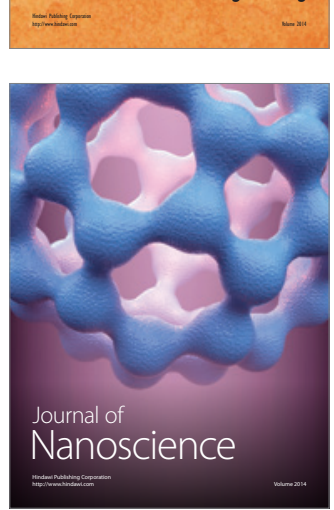
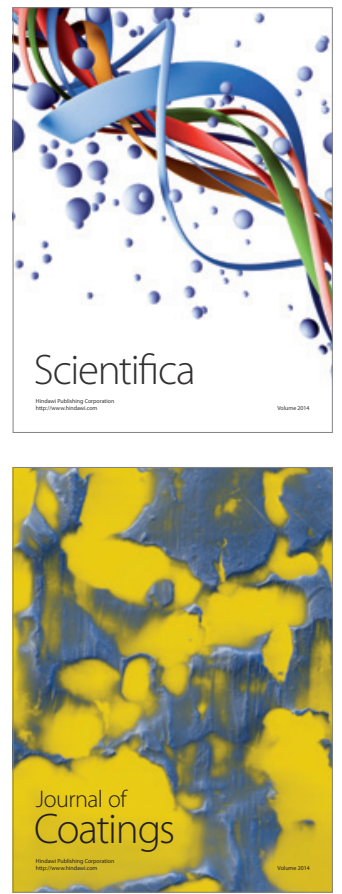
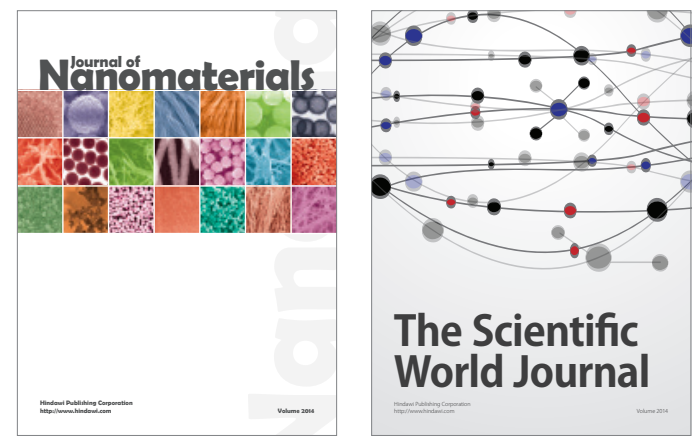

The Scientific World Journal
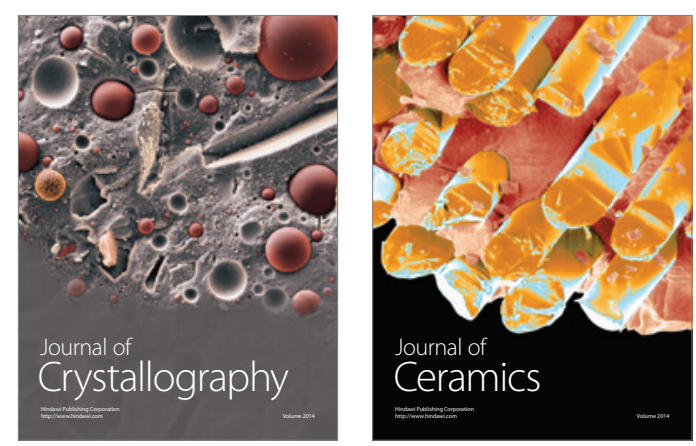
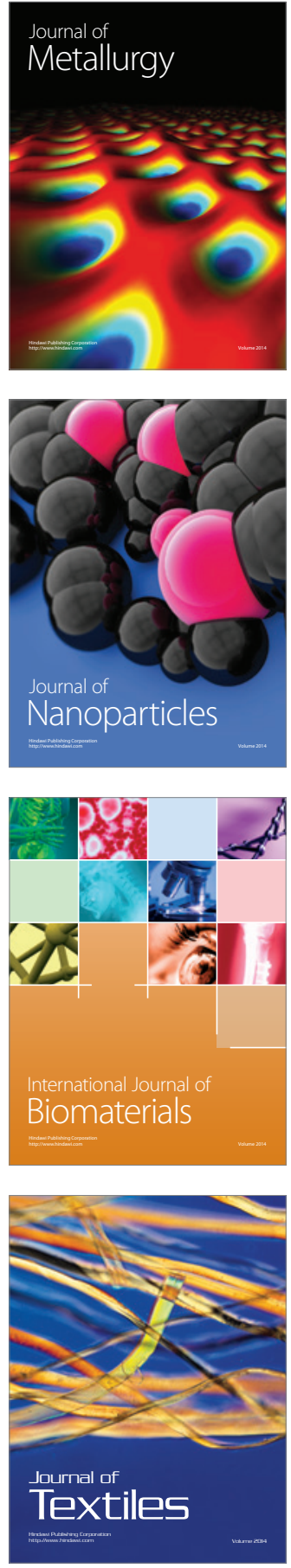